\title{
Erratum to: Classification of Cataract Fundus Image Based on Retinal Vascular Information
}

\author{
Yanyan Dong ${ }^{1}$, Qing Wang ${ }^{2}$, Qinyan Zhang ${ }^{1}$, and Jijiang Yang ${ }^{2(\bowtie)}$ \\ 1 Automation School, Beijing University of Post and Telecommunications, \\ Beijing, China \\ dyy0506@bupt.edu.cn, zh_qinyan@163.com \\ ${ }^{2}$ Research Institute of Information Technology, Tsinghua University, Beijing \\ 100084, China \\ qing.wang@tsinghua.edu.cn, yangjijiang@tsingha.edu.cn
}

\section{Erratum to: \\ Chapter "Classification of Cataract Fundus Image \\ Based on Retinal Vascular Information" in: \\ C. Xing et al. (Eds.): Smart Health, LNCS 10219, https://doi.org/10.1007/978-3-319-59858-1_16}

The initially published version of authors' affiliations was incorrect.

The updated online version of this chapter can be found at

https://doi.org/10.1007/978-3-319-59858-1_16 